\title{
LXXI. Memoir on the different compounds formed by the re-action of sulphuric acid on camphor
}

\section{Chevreul}

To cite this article: M. Chevreul (1811) LXXI. Memoir on the different compounds formed by the re-action of sulphuric acid on camphor, Philosophical Magazine Series 1, 37:158, 425-436, DOI: $10.1080 / 14786441108563313$

To link to this article: http://dx.doi.org/10.1080/14786441108563313

曲 Published online: 18 May 2009.

Submit your article to this journal $\sqsubset \pi$

Џ Article views: 2

Q View related articles $\sqsubset$ 
for a long time unattended by rain, when the nights are clear, and small meteors, called falling stars, are numerous; when, I say, these circumstances happen together, I have observed that the bells of this column always ring with very irregular pulsations; and further, when rain succeeds such kind of weather, it commonly happens that their pulsations become weak, or cease altogether, and the bells become silent: on the contrary, wben the weather is fair, and when only diurnal cumuli prevail, they usually pulsate regularly. An ingenious metcorologist suggested to me, the other day, that the irregular pulsation of the bells might be occasioned by the electric fuid's passing downward to the earth in pulsations, which might be the case when it was very irregularly distributed in the atmosphere.

To me it appears that this irregular distribution of the electric fluid would be indicated by the multiform appearance of the cirrus cloud which I have described, for the particular office of this cloud seems to be that of serving as a conductor of electricity. The same circumstance would also give rise to the occasional appearance of other modifcations. All however that can be said on this interesting subject at present is, that there seems to be a connection worth attending to between the kind of action of the column, and the kind of weather which prevails, indicated by the various and peculiar appearances of the clouds. Future observations may lead to the knowledge of adjunct circumstances which may have their share in producing these changes. To engage the co-operation of other meteorologists, by which alone the science cau be brought to any degree of perfection, is the object I have in view in soliciting the favour that this may be inserted in your Philosophical Magazine. I remain, sir, Your constant reader and servant,

St. Helea's Place, June 14, 1811.

Thomas Forstek.

LXXI. Memoir on the different Compounds formed by the Re-action of Sulphuric Acid on Camphor. By M. CHEVREUL**.

$\mathrm{H}$ Iaving shown in my two furmer memoirs, that tanning substances, formed by the re-action of nitric acid upon many vegetable compounds, were combinations of that acid with different substances, the greater part of which are unknown

- Annales de Chimie, tome bx. 
to us; it remains for me to inquire, if the third species of tannin of Mr. Hatchett (formed by the sulphuric acid) is a combination analogous to the others. I directed my researches to the tanning matter obtained from camphor, because it is that which particularly engaged the attention of Mr. Hatchett. Although I do not think that all the immediate principles of vegetables, when brought into contact with sulphuric acid, would be acted upon precisely in the same manner as camphor, I yet have reason to believe, that the results presented by this last are applicable to those substances which have a similar composition. The sulphuric acid, in its mode of acting, appears in general to possess more uniformity than the nitric acid does. The experiments which I am going to relate, were made upon camphor that was perfectly pure.

I put into a retort, to which a balloon was adapted, thirty grammes of camphor and sixty of pure sulphuric acid. The mixture became dark and yellow. I exposed it to a gentle heat for two hours. A great deal of sulphurous acid was disengaged. I then poured upon the thick brown liquor remaining in the retort, sixty grammes of sulphuric acid, and distilled. There came over into the receiver weak sulphuric acid, sulphurous acid, and a yellow volatile oil having a strong smell of camphor. Towards the end of the operation there was produced a small quantity of sulphuretted hydrogen, which was decomposed by the sulphurous acid; there was then scarcely any liquor left in the retort.

The residue, after distillation, was washed in boiling water until the washings werc no longer sensibly coloured. The first washings contained an excess of sulphuric acid; the others scarcely contained enough to be sensible to barytes. I shall first begin with the examination of the coaly residuum, and shall afterwards treat of the matters contained in the washings.

\section{Action of Water.}

The coaly residuum was of a bright black colour; its taste was a little acid, upon its being held some time in the mouth; it slightly reddened turnsole paper moistened with water. When boiled some hours in distilled water, the water was scarcely at all tinged. The water thus boiled upon the matier, formed no precipitate with nitrate of barytes and acetate of lead, even after standing twenty-four hours. Evaporated to dryness, there remained a trace of brownish colour, which became red on the application of barytes water. These trials convinced me there was no longer 
longer any uncombined sulphurous acid in this coaly residuum, and that, if it contained any, it must have been in a state of close combination.

\section{Action of Heat.}

I distilled four grammes of the coaly residuum in a small retort provided with a balloon, and obtained the following products; 1 . a little moisture; 2. sulphurous acid ; 3 . sulphuretted hydrogen, part of which was decomposed by the sulphurous acid, and part remained in the balloon, although there was in it an excess of the acid; 4. a red oil, which became brown on exposure to the air. This product, some time after its distillation, gave out an aromatic sulphurous odour resembling that of amber and pyritous coal; saturated with potass, it gave out volatile alkali, but in too small a quantity to enable me to draw any inference from its presence.

I observed in another experiment, made over mercury in a very small apparatus, that it gave out carbonic acid gas, oxy-carburetted hydrogen gas, sulphuretted hydrogen gas, and much less sulphurous acid than in the former distillation, which was made into a vessel containing a large quantity of atmospheric air. The action of heat proves, that besides carbon and hydrogen there are contained in the coaly residuum sulphur and oxygen, but it does not determine whether these last exist in it in the state of sulphuric acid, or are immediately combined with the carbon and hydrogen. However, if we consider that the coaly residuum reddens turnsole, the first opinion is the most probable, and $I$ adopt this as being the one most consistent with the facts and with analogy*.

The matter remaining in the retort after the distillation of the remainder of the coaly substance was black, semifused, formed of small shining grains, and resembled coak; it weighed two grammes and two deci-grammes. It was inodorous; but, on being exposed to the air for some time on a plate of copper, it blackened that metal, and gave out a smell of sulphuretted hydrogen. This phæmomenon induced me to make some experiments upon this substance, in order to ascertain in what state the sulphur existed in it. I boiled it in water, but this did not dissolve the least particle of it; it caused no precipitate either with water of barytes, or with a solution of acetate of lead. I boiled it

* Yet it is not impossible but there may be in this compound a small quantity of sulphur in a combustible state. 
several hours in a solution of potass, and the result was the same.

Not being able by these methods to detect the presence of sulphur in this substance, I thought of detonating it with nitrate of potass. The residuum of the detonation, dis. solved in water, filtered and supersaturated with nitric acid, afforded by the addition of nitrate of barytes an abundant precipitate of the sulphate. These experiments show that sulphur may be so intimately combined with carbon as to resist the action of a red heat, and of liquid potass. We cannot admit the presence of sulphuric acid in this combination; for all the known facts prove that the compounds in which this acid is the most concentrated do not resist; at a high temperature, the affinities of carbon and hydrogen. If oxygen at all exist in this combination, it can only be in a very small proportion, since it does not by heat act upon the combustible substances, so as to form with them gaseous products. From all these considerations, it appears to me, that we ought to regard the matter remaining after the distillation of the coaly residuum as a combination of carbon, sulphur, and a small quantity of hydrogen.

The solid combination of carbon and sulphur is not a fact entirely unknown to chemists. MM. Clément and Desormes have spoken of it in their Memoir upon Carbon; since which time M. Amédée Berthollet has shown, that by passing sulphur in a state of vapour over charcoal, a certain portion of the sulphur becomes fixed in it. The analysis of gunpowder yields the same combination. When the nitrate of polass has been carried off by means of water, the residuum, which is not soluble, and which consists of charcoal and sulphur, parts but with a vcry small quantity of this latter, by the action of heat; and to this is owing the sulphuric acid that is formed when the residumm already heated in a close crucible comes to be burned. These interesting facts on the analysis of gunpowder were communicated to me by M. Proust, when I gave him an account of my labours.

M. Proust, much struck with the odour of sulphurous acid generally exhaled from pit-coals towards the end of their combustion, thought at first that the sulphur in them had formed with the carbon a combination analogous to what I have described; but having afterwards observed that these coals plunged into nitric acid burned to the last without exhaling the least odour of sulphurous acid, he at length concluded that the sulphur was not combined with carbon, but with iron. I mention this fact, because it ag- 
pears to me to be of great importance, as I shall show herehfter.

\section{Action of Potass.}

Having in a former experiment observed that a mixture of ten parts of saturated carbonated potass with two parts of the coaly residuun exhibited scarcely the slightest traces of sulphuric acid, I wished to know if pure potass would have any geater effect. For this purpose, I boiled for two hours two grammes of the coaly residuum in water containing six grammes of pure potass dissolved in it. I left the substances to react on each other for the space of 12 hours afterwards. I then poured off the water and filtered it. A brown liquid ran through, and there remained upon the paper a black matter, which was washed in boiling water until the water was no longer coloured, and no longer gave any precipitate with water of barytes. The filtered alkaline solution was saturated with nitric acid *, there was a slight effervescence, a precipitation of brown floccules took place, and the liquid lost its colour. This liquor being again filtered, there was added to it a solution of barytes; at first there was not any precipitation, but in about two hours it threw down a very small particle of sulphate. Hence it follows, that the potass subtracts from the coaly residuum but an infinitely small quantity of sulphuric acid.

The brown floccules, which had been precipitated from the alkaline liquor by means of the nitric acid, were washed, first in cold and afterwards in warm water: this last dissolved a portion of them, and without doubt would have dissolved the whole, had it been in sufficient quantity. This solution was slightly acid: sulphuric, nitric, and muriatic acids caused a precipitation in it, probably by uniting with the matter it held in solution; barytes produced from it a precipitate soluble in bot nitric acid. I had too small a quantity of the brown floccules dissolved in potass to determine the nature of them, but I believe them to be composed of coaly residuum, nitric acid, and a small quantity of potass.

Part of the coaly residuum which had not been dissolved in the alkali, being well washed and dried, gave out when heated in a glass vessel, 1 . carbonic acid gas in great quantity; 2. sulphuretted hydrogen gas, 3. oxy-carhuretted hydrogen gas; 4. a coal which gave out a very strong smell of sulphur when breathed upon. I supposed from this cir-

* An excess of acid is necessary to render the precipitation complete. It would appeat that this excess enters into combination.

cunistance, 
cumstance, that it might retain some sulphuret of potass, and $I$ washed it in boiling water; but if the water dissolved any of it, it was too small a quantity to be rendered sensible by the acetate of lead, which did not blacken the liquor. After these results, I adopted another method of analysis; I burned the coal and obtained a grayish cinder, which being washed in water afforded some carbonate and some sulphate of potass, discoverable by the solutions of barytes and of platina. As, therefore, there were in this compound some sulphur and some potass, not soluble in hot water, it is very natural to conclude, that these substances are fixed in the coaly matter by a real chemical affinity. From all these circumstances, I am of opinion, that one part of the alkaline and earthy bases found in the coals of wood, \&c. may exist in them in a state of strict combination,

\section{Action of Nitric Acid.}

Five grammes of the coaly residuum were put into a re tort with fifty grammes of nitric acid heated to $32^{\circ}$. Red vapours and carbonic acid were immediately disengaged. On applying heat, the action became much stronger, and the liquor foamed greatly. When the greatest part of the liquid had passed over into the receiver, I poured twentyfive grammes more of the acid into the retort: a complete solution took place, and the liquor was of a deep yellow orange colour: when concentrated in the retort, $\mathbf{I}$ poured it into a glass of water; there was precipitated a deep yelJow flocky matter; this was separated by the filter, and washed with a large quantity of cold water. All the waters were mixed and evaporated to dryness, so as to drive off in a great measure the excess of acid. The residue was treated by a smaller quantity of water; by these means, there was procured a small portion of yellow floccose matter, partly soluble. I first examined the matter dissolved in the water, and then the yellow flocky substance.

The aqueous solution, separated from its excess of acid, and from the matter partly soluble, by several successive evaporations and solutions, was of a brown-yellow colour; its taste was acid, bitter, and astringent; yellow floccules were precipitated from it by gelatin. It contained a little sulphuric acid, discoverable by barytes; it is true, there was a flaky precipitate formet by the astringent substance, but this was soluble in nitric acid. (I believe that this sul-

* Centigrade Thermometer. 
phuric acid proceeded from a portion of the coaly residuum, which had been completely destroyed by the nitric acid; for out of four experiments I made, two of them afforded me not the least trace of sulphuric acid; the precipitate formed by barytes was entirely soluble in nitric acid.) In order to separate the uncombined sulphuric acid, I boiled the astringent liquor which contained it with carbonate of barytes*; I then evaporated it to dryness, and treated the residue with water: there remained a small quantity of sulphate of barytes mixed with a little of the compound of astringent matter and barytes. The aqueous solution contained the astringent matter pure, or at least separated from the sulphuric acid. I evaporated this solution to dryness, and distilled the residue in a small glass retort"with a balloon adapted to it. There came over, 1st, a yellow liquor of an acid and bitter taste,-I thought this was nitric acid; 2. sulphurous acid, which was ascertained by its odour, and by the combination it formed with oxide of copper ; 3. sulphuretted hydrogen gas ; 4. sulphur, proceeding from the decomposition of the sulphurous acid and sulphuretted hydrogen; 5 . a thick brown-oily liquor, mixed with prussic acid and ammonia; 6 . some small needle-like crystals, whose nature I could not determine, on account of their small quantity; 7. a very puffy coal, containing ammonia, which was discoverable by the smell, and by a rod impregnated with weak nitric acid $\uparrow$. This coal contained a little sulphur; for, when burned with nitrate of potass, it afforded some sulphate by means of nitrate of barytes.

It follows from these facts, that the astringent matter, soluble in water, produced by the re-action of the nitric acid upon the coaly residuum, is formed of nitric acid, sulphuric acid, and the coaly matter.

I come now to the examination of the yellow substance, partly soluble in water. I boiled it in water until the washings no longer precipitated bary tes in the form of sulphate. The matter thus washed was of an orange-yellow colour, it had an acid and bitter taste, it slightly reddened turnsole paper; heated in a glass tube, it quickly melted, disengaging an aromatic odour, a little sulphureous; boiled for several hours in water, it diffused a very strong smell of musk, and by distilling the water a liquor was obtained

* The liquor digested twenty-four hours upon litharge, equally lost its nulphuric acid, but the carbonate of barytes appeared to me preferable.

$t$ I think this ammonia was formed when the hot coal came into contact with the air.

impregnated 
impregnated with the same ndour. The water which was boiled upon this substance was of a yellow colour and slightly acid, as appeared by the test of turnsole: with barytes water, it formed a precipitate of vellow floccules entrrely soluble in nitric acid. Although this substance was boiled in a very large proportion of water, the latter was alwaye tinged of a yellow colour. After being thus washed, it was subjected to the action of heat in a glass vessel; it melted, and there were obtained, among other products, nitrous vapour, nitrous gas, and a coal which had a sulphureous smell. Although 'I only operated on two decigrammes of this substance, $I$ am led to believe that it is composed of nitric acid, a small quanity of sulphuric acid, and of a carlonated matter more hydrogenated than that which forms the coaly residuum*.

The artificial production of a substance which possessed the odour of musk had already been observed by Geoffroy in $3726 t$, and by Margraff in 1758t. The first discovered it upon mixing sulphuric and nitric acids with clear oil of petroleum; the second, in mixing rectified oil of amber with nitric acid. I myself have often had occasion to remark the same thing with the resinous substance which remains after the inflammation of oil of turpentine with the sulphuric and nitric acids. It appears, that in all these instances it is formed by the combination of nitric acid, and sometimes of sulphuric acid, with the oily matter.

The results of the action of nitric acid upon the coaly sesiduum offer the following interesting facts to our consideration.

1. They prove that the coaly residuum which contains sulphuric acid does not part with it upon being combined with nitric acid, (except the small quantity of it which is loose;) and that, if we rely upon the test of barytes to demonstrate the absence of sulphuric acid, we shall be deceived, because the precipitate in which we should suspect the acid to be would be soluble in nitric acid.

2. They confirm what I advanced in a former memoir, on the combination of sulphuric acid with the tanning matter of pit-coal, which forms with barytes a compound soJuble in nitric acid.

3. They show that, by the re-action of nitric acid upon a coaly substance containing a great deal of hydrogen, a

* Having by chance left a certain quantity of this substance exposed to the rays of the sun, I observed at the end of two month that it was covered with small brilliant crystals.

$t$ Mernoirs of the Academy of Sciences.

$\$$ Opuscales, tome ii.

portion 
portion of the hydrogen and of the carbon may combine with the nitric acid, and form a compound, fusible by heat, and partly soluble in water, and which possesses some of the properties of resins; and that, consequently, we are perbaps too hasty in regarding pit-coal, which furnishes similar products when treated with nitric acid, as being formed of a resin similar to that of recent vegetables, and of a coaly substance: this opinion may be true, but the facts brought in support of it are not sufficient to prove it.

4. They demonstrate, that, if the manner in which the coaly residuum is acted upon by the nitric acid indicates some analogy between this compound and pit-coal, yet the facts related above do not permit us, at least at present, to regard them as having one and the same origin. Indeed when the sulphuric acid acts upon the camphor, (and what takes place with that must take place with other vegetable substances, ) it unites with the uncombined carbonated substance: consequently, if pit-coal was formed in the same tnanner, we must find sulphuric acid in it: besides, if we recollect that $M$. Proust, although he suspected there was a combination of sulphur with carbon in pit-coal, could not discover the least trace of sulphurous acid in that substance, when deprived of its sulphate of iron, we shall consider the hypothesis of the formation of pit-coal, by means of the sulphuric acid, as totally inadmissible.

I wished to determine the quantity of sulphuric acid contained in 100 parts of coaly residuum. For this purpose, I fused nitrate of potass in a crucible of platina, and projected into it the residuum in small quantities: although I had taken every necessary precaution that nothing should be lost, I always found that a small portion of the matter escaped combustion, and that it was carried out of the crucible by the gas, which was disengaged with impetuosity : the quantities of sulphuric acid here given, must not, therefore, be taken as completely accurate. In one experiment, I obtained a precipitate of sulphate of barytes, which indicated 5 parts of sulphuric acid; and, in another, a precipitate, which indicated 6 parts in the 100 of the coaly residuum.

One circumstance I must not omit to mention, - that I discovered traces of the existence of lime and oxide of iron in the camphor I employed in my experiments, and which had been twice sublimed.

Examination of the Washings.

These were, as I have mentioned, divided into two parVol. 37. No. 158. June 1811. Ee cels; 
cels; the first did not differ from the others, only in cons taining an excess of sulphuric acid. That this excess of acid might not deccmpose the vecetable matter in solution, I only reduced the liquor to one-fourth of its original quantity. In this state, it appeared green by refiction, and a yellowish red by refraction; (a phænomenon similar tn what takes place in the solution of ncphritic wood, with this difference, that the latter appears blue by reflection;) it precipitated gelatin, whence it is evident that it contained the tanning substance of Hatchett. To separate this latter from the sulphuric acid, I saturated it with barytes water. The sulphate was precipitated, and the combination of the astringent matter and barytes remained in the liquor: this was of a fine red colour: when muriatic acid was poured upon it to saturate the barytes, it regained the property of reflecting green, and parted with the rose colour : thus saturated, it precipitated gelatin; the combination of astringent matter and barytes, being dried and afterwards heated in a glass tube, was converted in to a sulphuret.

The second parcels of water contained an exceedingly small quantity of sulphuric acid: to separate this, I poured into it a few drops of the combination I have mentioned, (of the astringent malter and barytes) and filtered it. The filtered liquor might be considered as a solvition of pure astringent matter. It was acid, and presented the same appearances to the light as the first washing when concentrated; it precipitated gelatin; evaporated to dryness, there remained a substance, from which, when subjected to beat, there were disengaged sulphuretted bydrogen gas and sulphurous acid. The astringent matter had undergone some alteration by this evaporation; for, treated' with water, it formed a solution, which no longer reffected a green colour, and which precipitated barytes in brown floccules. This precipitate was soluble in nitric acid, and was converted into a sulphuret by calcination.

I regard the astringent matter as being composed of sulphuric acid, and a certain substance which at present is unknown to me. It is true, I have not been able directly to separate the sulphuric acid; but as I cannot detect in it either sulphuretted hydrogen or sulphurous acid, and as on the other hand it reddens turnsole, and contains oxygen and sulphur, the former opinion appears to me in the present state of science to be the most probable.

\section{Recapitulation.}

When sulphuric acid is distilled from camphor, therc are obtained, 
obtained, 1. a volatile vil*, having the smell of camphor: 2. a coaly residuum, which is a combination of sulphuric acid, and highly hydrogenated carbon: 3. an astringent matter, which is also a combination of sulphuric acid, but which seems to differ from the former in two respects, viz. that the matter combined with the acid is more hydrogenated, and that the acid is in greater proportion.

The coaly residuum is not perceptibly soluble in water; it appears, however, to impart to it an exceedingly small quantity of astringent matter.

By distillation it affords sulphuretted hydrogen, sulphurous acid, and carbonic acid; the residuum is a combination of carbon and sulphur. This last combination is formed as often as sulphur meets with carbon strongly heated: thus, charcoal over which the vapour of sulphur has been passed; the mixture of charcoal and sulphur, (proceeding from the analysis of gunp:swuer, when heated in a crucible, are compositions of this sort. It is very probable something of the same kind takes place in the decomposition of the sulphates by charcoal.

Potass takes up but a very inconsiderable portion of sulphuric acid from the coaly residuum: when these two substances are boiled together, two compounds are formed, one soluble, with an excess of alkali, and the other insoluble, with an excess of the coaly residuum. Nitric acid dissolves it totally, and forms with it two substances; one very soluble in water, which precipitates gelatin, and which forms with barytes a compound soluble in nitric acid, although it contains sulphuric acid; the other compound partly soluble, which fuses by heat, and gives out nitrous acid; this appears to be more hydrogenated than the former one.

Although the coaly resilunm possesses some properties similar to those of pit-coal, the ahsence of sulphur and of sulphuric acid in this last, will not permit us to regard them as having a common origin. The analogous properties of these two substances appear to belong in general to all coaly substances which contain much hydrogen.

The astringent matter is soluble in water. Its solution appears green by reflcction, and red by refraction: it precipitates gelatin; it is acid, and by distillation it affords sulphuretted hydrogen and sulphuric acid. It forms with

* I much regret not being able to examine the nature of tilia product. It would be interesting to know if it contains sulphuric acid and camphor. By distilling it with pistiss, 1 obtained an odorous crystallized sublimate, but it was in too small a quantity to enable me to determine exactly its nature. 


\section{On the fundamental Property of the Lever.}

barytes a compound soluble in water; it undergoes some alteration while the solution is evaporating; it appears that the sulphuric acid combined with it, re-acts upon the vegetable matter and blackens it. The sulphuric acid cannot be separated from the astringent matter without decomposing it.

In the analysis of vegetables, we are not to conclude that whenever any substance precipitates gelatin, that substance is tannin; for it is very probable, that there are several substances very different from each other, which possess that property. Lastly, since we find, 1st, that for the most part, those substances which form a precipitate with gelatin are acid; 2d, that frequently this animal matter cannot be precipitated from vegetable infusions, without the addition of an acid; and, $3 \hat{d}$, that the greatest number of natural tannins redden turnsole; we may fairly presume that tannins are combinations of vegetable acids with substances of various natures.

LXXII. Demonstration of the fundamental Property of the Lever. By David Brewster, LL.D. F.R.S. Edin.*

I all the attempts of the most eminent modern mathematicians, to obtain a simple and satisfactory demonstration of the fundamental property of the lever, the solution of this problem given by Archimedes should still be considered as the most legitimate and elementary. Galileo, Huygens, De la Hire, Sir Isaac Newton, Maclaurin, Landen, and Hamilton, have directed their attention to this important part of mechanics; but their demonstrations are in general either tedious and abstruse, or founded on assumptions too arbitrary to be recognised as a proper basis for mathematical reasoning. Even the demonstration given by Archimedes is not free from objections, and is applicable only to the lever, considered as a physical body. Galileo, though his demonstration is superior in point of simplicity to that of Archimedes, resorts to the inelegant contrivance, of suspending a solid prism from a mathematical lever, and of dividing the prism into two unequal parts, which act as the power and the weight. The demonstration given by Huygens assumes as an axiom, that a given weight removed from the fulcrum has a greater tendency to turn the lever

* From the Transactions of the Royal Sociefy of Edinburgh. 